\title{
Clinical Evaluation Of Evolocumab For The Treatment Of Homozygous Familial Hypercholesterolemia In Chinese Patients
}

This article was published in the following Dove Press journal:

Therapeutics and Clinical Risk Management

\author{
Chin-Chou Huang $\mathbb{1 D}^{1-5}$ \\ Min-Ji Charng $\mathbb{1 D}^{2,3}$ \\ 'Department of Medical Education, Taipei \\ Veterans General Hospital, Taipei, \\ Taiwan; ${ }^{2}$ Division of Cardiology, \\ Department of Medicine, Taipei Veterans \\ General Hospital, Taipei, Taiwan; ${ }^{3}$ Faculty \\ of Medicine, School of Medicine, National \\ Yang-Ming University, Taipei, Taiwan; \\ ${ }^{4}$ Cardiovascular Research Center, \\ National Yang-Ming University, Taipei, \\ Taiwan; ${ }^{5}$ Institute of Pharmacology, \\ National Yang-Ming University, Taipei, \\ Taiwan
}

\begin{abstract}
Evolocumab, which can lower low-density lipoprotein (LDL) cholesterol levels by approximately $60 \%$ and prevent cardiovascular events in patients with cardiovascular disease, is a monoclonal antibody that inhibits proprotein convertase subtilisin/kexin type 9 (PCSK9). Some studies have investigated its efficacy and safety in the treatment of the homozygous form of familial hypercholesterolemia (HoFH), and others have focused on its efficacy and safety in Asians with high cardiovascular risk. Although no direct evolocumab clinical trials have been conducted in Chinese HoFH patients, its efficacy and safety in the Chinese population should be similar to those in other ethnic groups.
\end{abstract}

Keywords: Chinese, evolocumab, homozygous familial hypercholesterolemia, low-density lipoprotein, proprotein convertase subtilisin/kexin type 9

\section{Introduction}

Familial hypercholesterolemia (FH; MIM\# 143890) is one of the most common hereditary metabolic diseases. Its incidence ranges from $1 / 200$ to $1 / 500$ individuals. $^{1,2}$ The prevalence of the heterozygous form of $\mathrm{FH}(\mathrm{HeFH})$ is reported to be about $1 / 200-250,{ }^{3}$ while that of the homozygous form $(\mathrm{HoFH})$ is reported to be about 1/160,000-300,000. ${ }^{4}$ However, previous FH studies were focused mainly on Caucasian populations; hence, a complete investigation of its frequency in the Chinese population does not exist.

Some of the lipid metabolism defects that have been observed in FH patients include reduced LDLR-mediated low-density lipoprotein (LDL) cholesterol catabolism, impairment of apolipoprotein B (APOB)-mediated LDL clearance, and increased proprotein convertase subtilisin/kexin type 9 (PCSK9) levels, which mediates the posttranslational destruction of LDLRs. ${ }^{5,6}$ The impairment in the functionality of LDLR, APOB and PCSK9 in HoFH patients results in a marked increase in LDL cholesterol levels and clinical cardiovascular events.

In a recent comparison of $\mathrm{FH}$ health care in Asia-Pacific and Southern Hemisphere countries, important gaps, including in detection and treatment, were still noticed across the FH care continuum, particularly in less economically developed countries. ${ }^{7}$ Thus, comprehensive strategies are needed to improve FH patient care in these countries.

PCSK9 is a key regulator of LDLR function. Evolocumab, a monoclonal antibody that inhibits PCSK9, prevents PCSK9-mediated LDLR degradation and
Chin-Chou Huang

Department of Medical Education, Taipei Veterans General hospital, No. 20I, Sec.

2, Shih-Pai Road, Taipei II217, Taiwan,

R. O. C.

Tel +886228757725

Fax +886228757726

Email cchuang4@vghtpe.gov.ytw 
permits LDLR to recycle back to the liver cell surface with higher abundance, thereby lowers LDL cholesterol level by approximately $60 \%$ clinically. ${ }^{8}$ The LDL-lowering effect of evolocumab in HoFH is heavily dependent on the residual function of mutated LDLR, which is defective if its residual activity is $2 \sim 30 \%$ of normal, and negative if it is less than $2 \%$. The efficacy of evolocumab in HoFH patient therefore was quite variable depending on the type of LDLR mutation and its residual activity. Previous studies on the evolocumab's efficacy and safety in HoFH patients focused mainly on Caucasian populations, which were lacking in Chinese population. Therefore, the objective of this study was to review current evidences of its use in the treatment of Chinese HoFH patients.

\section{Evolocumab's Efficacy And Safety In HoFH}

Evolocumab's efficacy and safety in HoFH treatment was first evaluated in an open-label, single-arm, multicenter, dose-scheduling pilot study consisting of $8 \mathrm{HoFH}$ patients (2, LDLR-negative; 6, LDLR-defective), who were on a stable drug therapy (TESLA part A, Table 1). ${ }^{9}$ They were treated with subcutaneous evolocumab $(420 \mathrm{mg})$ every 4 weeks for 12 weeks, followed by the same dose treatment every 2 weeks for an additional 12 weeks. The mean change from baseline in LDL cholesterol level at week 12 was $-16.5 \%$ and $-13.9 \%$ with the 4 - and 2 -week dosing, respectively. Over the treatment periods, no reduction in LDL cholesterol was observed in the 2 LDLRnegative patients, while in the 6 LDLR-defective patients, the reductions were $19.3 \pm 16 \%$ and $26.3 \pm 20 \%$ with the 4- and 2-week dosing, respectively ( $p=0.0313$ for both treatment periods), and ranged from $4 \%$ to $48 \%$ with the 2-week dosing. There were no serious side effects. This study, which was a very early stage study of the use of evolocumab in the treatment of HoFH patients, demonstrated a significant and dose-related lowering of LDL cholesterol with evolocumab use in LDLR-defective HoFH patients, while no reduction was observed in those who were receptor negative.

The TESLA part B study was a randomized, doubleblind, placebo-controlled evolocumab phase III trial undertaken at 17 sites in 10 countries in North America, Europe, the Middle East, and South Africa (Table 1). ${ }^{10}$ Fifty eligible HoFH patients (age, $\geq 12$ years), who had been on stable lipid-regulating therapy for at least 4 weeks, and had not received lipoprotein apheresis, were randomly allocated in a $2: 1$ ratio to receive subcutaneous evolocumab $(420 \mathrm{mg})$ or placebo every 4 weeks for 12 weeks. Of the 50 eligible patients who were randomly assigned to the 2 treatment groups, 49 actually received the study drug and completed the study (16 in the placebo group and 33 in the evolocumab group). Compared with the placebo, evolocumab significantly reduced LDL cholesterol levels at week 12 by $30.9 \%$ ( $95 \% \mathrm{CI},-43.9 \%$ to $-18.0 \%$; $p<0 \cdot 0001)$. Treatment-induced adverse events occurred in $10(63 \%)$ of the 16 patients in the placebo group, and in $12(36 \%)$ out of the 33 in the evolocumab group. No serious clinical or laboratory adverse events occurred, and no anti-evolocumab antibody development was detected during the study.

The TAUSSIG study was an open-label, non-randomized evolocumab phase III trial, which was undertaken at 35 sites in 17 countries. ${ }^{11}$ In the interim subset analysis, $106 \mathrm{HoFH}$ patients (aged $\geq 12$ years), who were on a stable LDL cholesterol-lowering therapy for at least 4 weeks were included, and they all received evolocumab (420 mg) subcutaneously once every month, or once every 2 weeks if they were on apheresis (Table 1). The dosing could be increased to every 2 weeks after 12 weeks in patients who were not on apheresis. The results revealed that after 12 weeks, mean LDL cholesterol decreased from baseline by $20.6 \%$, and was maintained until week 48 . In 47 out of the 72 patients who were not on apheresis at study entry, increasing evolocumab dosing to every 2 weeks led to an additional mean LDL cholesterol decrease of $8.3 \%$. In a post-hoc analysis, mean reductions in LDL cholesterol in patients on apheresis were significant at week $12(p=0.0012)$ and week $48(p=0.0032)$, and did not differ from reductions observed in patients who were not on apheresis at week $12(p=0.38)$ and at week $48(p=0.09)$. The interim results suggested that evolocumab is well tolerated and is an effective additional option for reducing LDL cholesterol in HoFH patients, with or without apheresis.

Currently, the TAUSSIG study has expanded to include a total of 300 subjects (106 with HoFH and 194 with severe HeFH, respectively), who received evolocumab for a median of 4.1 years (Table 1). ${ }^{12} \mathrm{~A}$ total of 61 subjects were on apheresis, and the median change in LDL cholesterol from baseline to week 12 was $-18.3 \%$ in those with $\mathrm{HoFH}$, and $-57.1 \%$ in those with severe HeFH. Of the 48 subjects who were uptitrated, the median change in LDL cholesterol improved from $-16.3 \%$ at week 12 to $-29.3 \%$ after 12 weeks of $420 \mathrm{mg}$ of Q2W. This study is the largest 


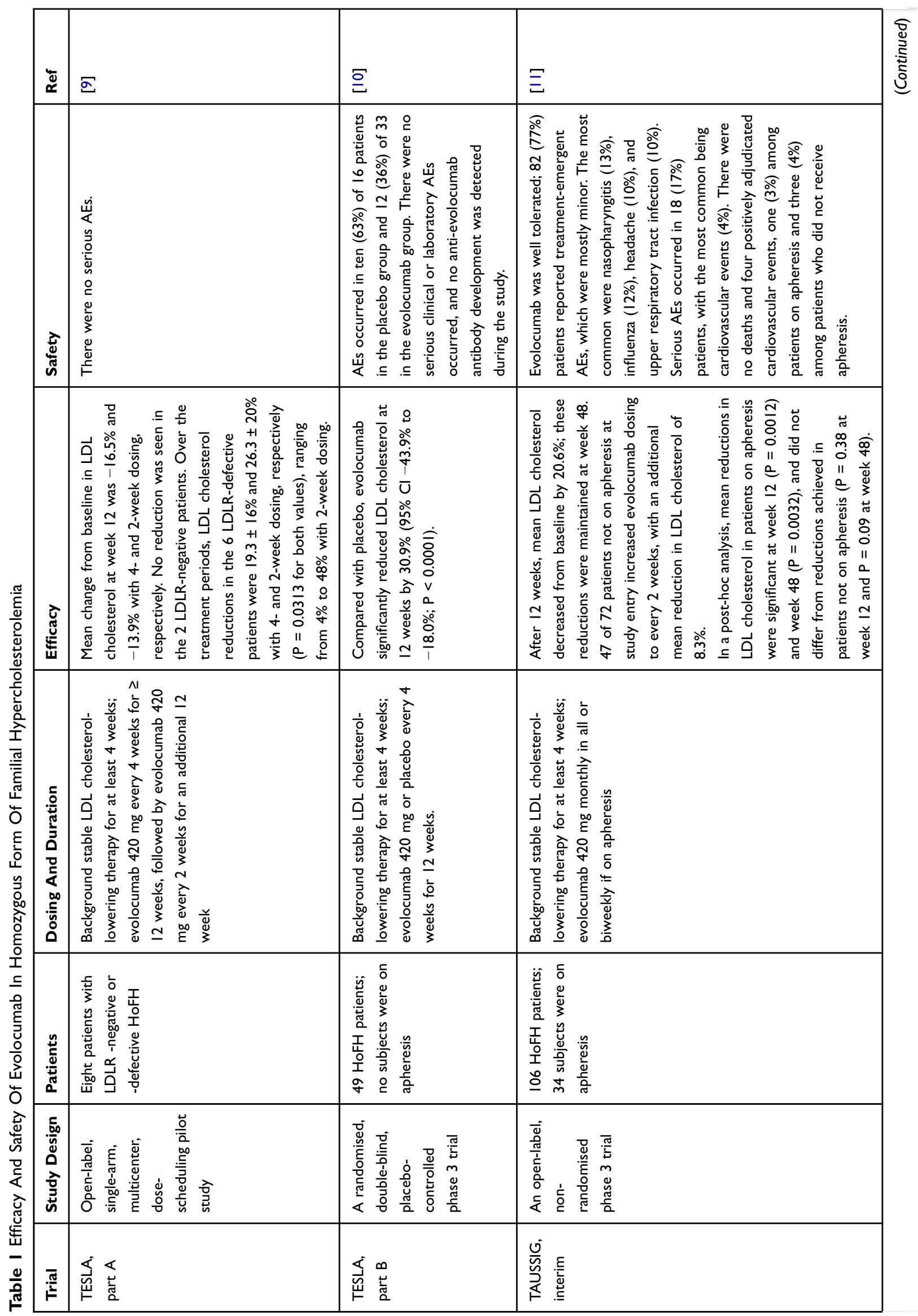




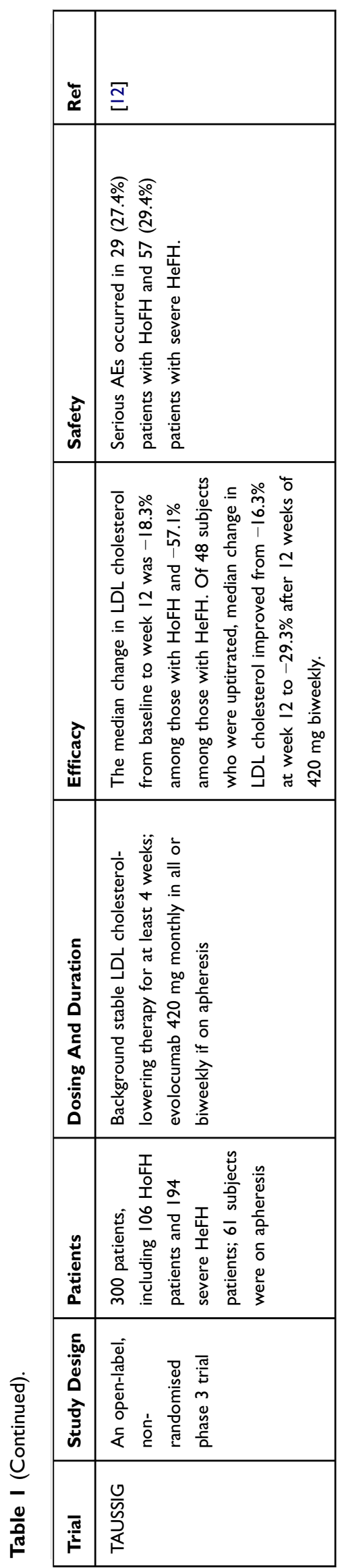

and longest on $\mathrm{HoFH}$, and its results also demonstrated that evolocumab is well tolerated, and effectively reduced LDL cholesterol over a median of 4.1 years. Although relative reductions of LDL cholesterol were lower in subjects with HoFH, absolute reductions were similar for both HoFH and severe HeFH patients.

\section{Evolocumab's Efficacy And Safety In The Asian Population}

There are only a few studies on evolocumab efficacy and safety of in the Asian population, including Japanese and Chinese subjects, and the subjects in these studies were those with high cardiovascular risk instead of those with familial hypercholesterolemia (Table 2).

In the YUKAWA study, the efficacy and safety of a 12 week evolocumab treatment was evaluated in high cardiovascular risk statin-treated Japanese patients. ${ }^{13}$ Eligible patients (310) receiving stable statin ( \pm ezetimibe) therapy were randomly placed in 1 out of 6 treatments groups: placebo biweekly or monthly, $70 \mathrm{mg}$ or $140 \mathrm{mg}$ evolocumab biweekly, and $280 \mathrm{mg}$ or $420 \mathrm{mg}$ evolocumab monthly. The mean changes in LDL cholesterol level vs placebo were greatest in the high-dose groups $(-68.6 \%$ and $-63.9 \%$ with $140 \mathrm{mg}$ biweekly and $420 \mathrm{mg}$ monthly dosing, respectively). The study also showed that evolocumab significantly reduced LDL cholesterol and was well tolerated during the 12-week study in high-cardiovascularrisk statin-treated Japanese hypercholesterolemia patients.

In the open-label extension (OLE) OSLER study, evolocumab efficacy and safety were evaluated in a pooled analysis of patients from the 12-week YUKAWA studies who were followed up for 1 year. ${ }^{14}$ YUKAWA-1 and YUKAWA-2 were conducted in high-cardiovascular-risk Japanese hypercholesterolemia patients on statin therapy. Patients completing these studies were eligible for an OLE study. At the OLE study entry, patients were re-randomized in a 2:1 ratio to receive evolocumab + standard of care (SOC) or SOC alone (OSLER-1, $420 \mathrm{mg}$ evolocumab monthly; OSLER-2, $140 \mathrm{mg}$ evolocumab biweekly or 420 mg monthly). At parent-study baseline (YUKAWA-1 or YUKAWA-2 patients continuing into OSLER), mean baseline LDL cholesterol (on statin) was $119.7 \mathrm{mg} / \mathrm{dL}$. Overall rates of adverse events were comparable between evolocumab + SOC and SOC alone. In the YUKAWA patients receiving evolocumab + SOC, mean LDL cholesterol reductions from parent-study baseline to the OLE study (1 year) were 69.1\% (OSLER-1) and 65.1\% 


\begin{tabular}{|c|c|c|c|c|}
\hline 馬 & $\underline{\underline{m}}$ & 守 & $\underline{\square}$ & $\Xi$ \\
\hline 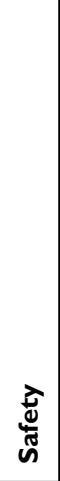 & 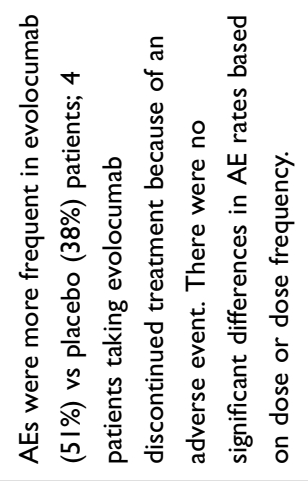 & 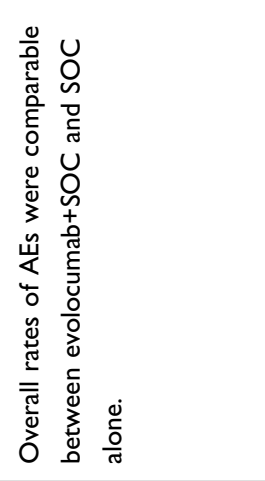 & 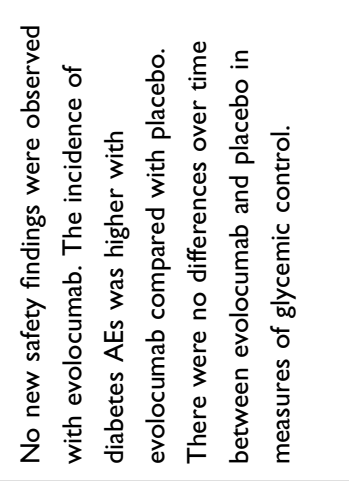 & 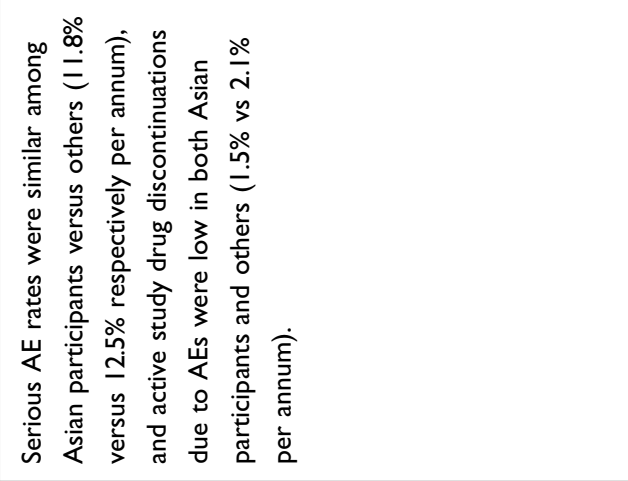 \\
\hline 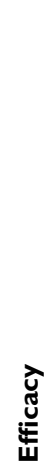 & 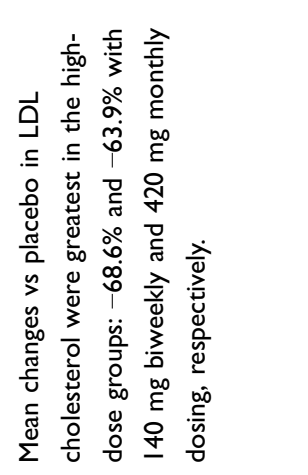 & 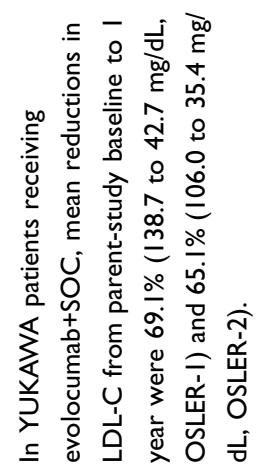 & 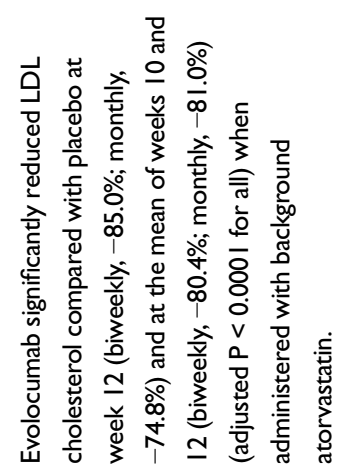 & 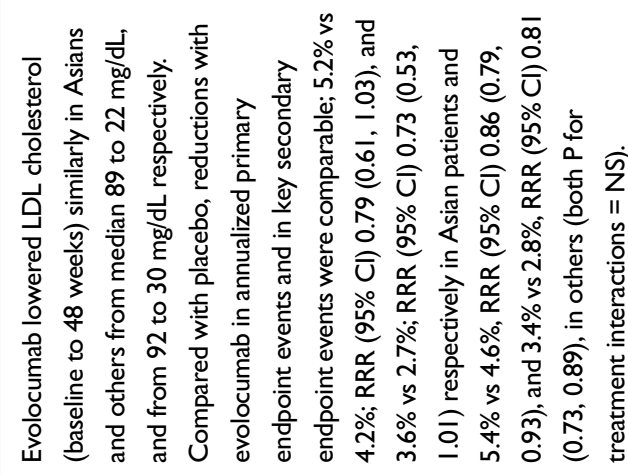 \\
\hline 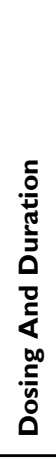 & 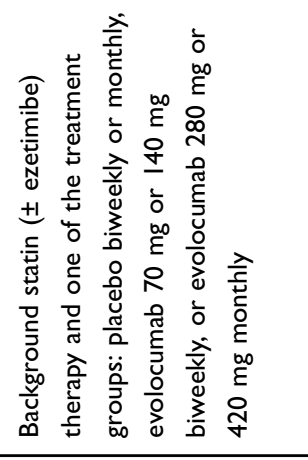 & 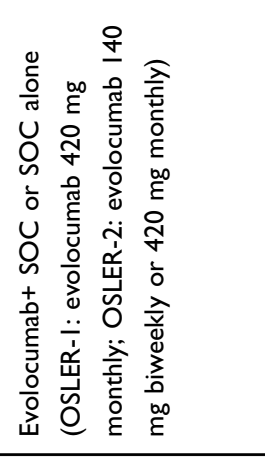 & 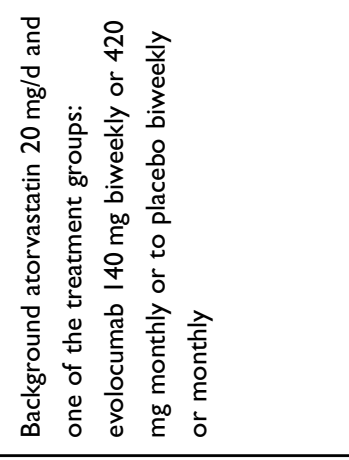 & 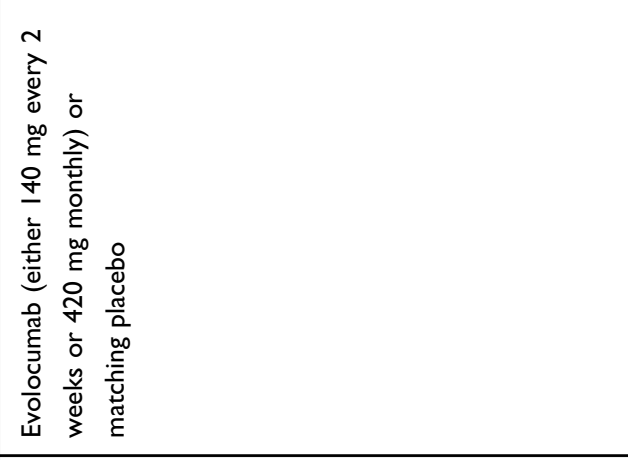 \\
\hline 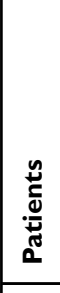 & 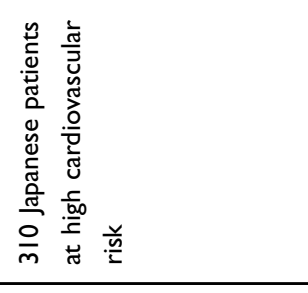 & 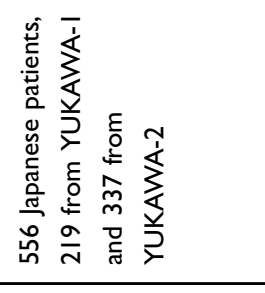 & 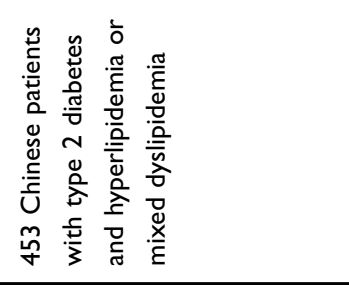 & 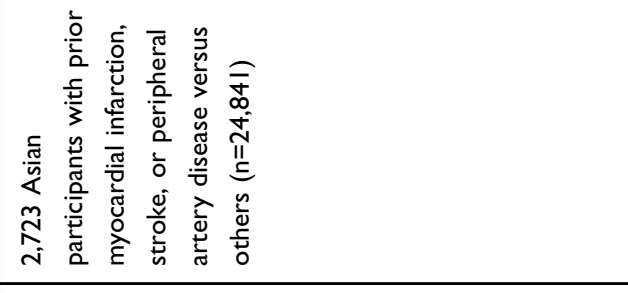 \\
\hline 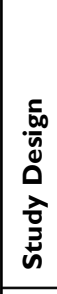 & 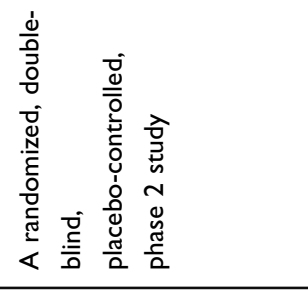 & 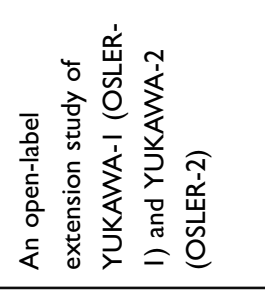 & 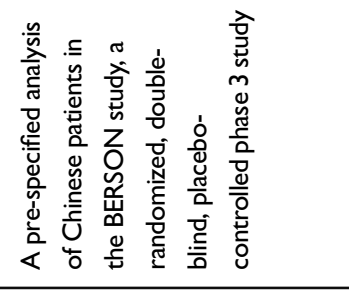 & 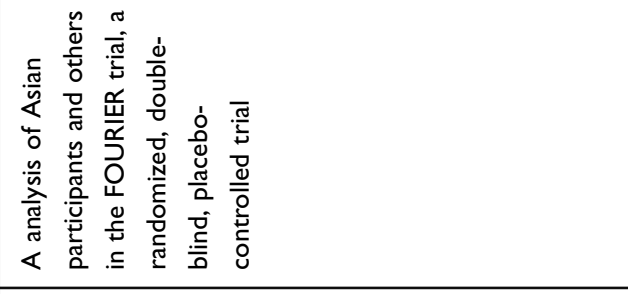 \\
\hline 趈 & $\begin{array}{l}\frac{1}{3} \\
\frac{\sqrt{3}}{2} \\
\frac{2}{2}\end{array}$ & $\begin{array}{l}\text { 山े } \\
\text { Оू }\end{array}$ & 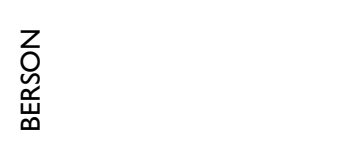 & 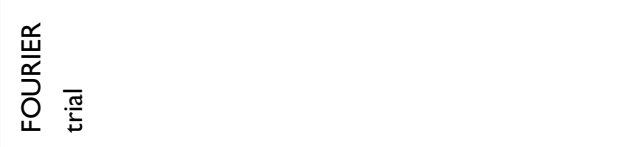 \\
\hline
\end{tabular}


(OSLER-2), showing that evolocumab was well tolerated and resulted in sustained LDL cholesterol reductions at 1 year.

The BERSON study was a double-blind, 12-week, phase III study conducted in 10 countries. ${ }^{15}$ Patients ( $\geq 18$ to $\leq 80$ years) with type 2 diabetes and hyperlipidemia or mixed dyslipidemia were enrolled. The patients were initiated with atorvastatin $(20 \mathrm{mg} / \mathrm{d})$, after which they were randomly divided in a $2: 2: 1: 1$ ratio to receive $140 \mathrm{mg}$ evolocumab biweekly, $420 \mathrm{mg}$ monthly, or placebo biweekly or monthly. In the pre-specified analysis of Chinese patients in the BERSON study in China, 453 patients were randomized, and 451 received at least one dose of the study drug (evolocumab or placebo). ${ }^{16}$ The results showed that evolocumab significantly reduced LDL cholesterol compared with the placebo at week 12 (biweekly, $-85.0 \%$; monthly, $-74.8 \%$ ) and at the mean of weeks 10 and 12 (biweekly, $-80.4 \%$; monthly, $-81.0 \%$ ) (adjusted $p<0.0001$ for all treated groups) when administered with background atorvastatin. The study also showed that evolocumab was well tolerated and significantly reduced LDL cholesterol and other atherogenic lipids in Chinese patients with type 2 diabetes and hyperlipidemia or mixed dyslipidemia, who were on statin. There was no notable impact on glycemic measures.

In the post-hoc analysis of the FOURIER trial, which included 27,564 participants, the effects of adding evolocumab (either $140 \mathrm{mg}$ subcutaneously every 2 weeks or $420 \mathrm{mg}$ subcutaneously monthly) versus matching placebo to background optimized statin therapy over a median 2.2 years follow-up on LDL cholesterol reductions, cardiovascular events and adverse safety events were compared, according to declared ethnicity (Asian, $\mathrm{n}=2,723$ vs Other $\mathrm{n}=$ 24,841). ${ }^{17}$ Evolocumab lowered LDL cholesterol (from baseline to week 48) similarly in Asians and others from a median of 89 to $22 \mathrm{mg} / \mathrm{dL}$, and 92 to $30 \mathrm{mg} / \mathrm{dL}$, respectively. Compared with placebo, reductions with evolocumab in annualized primary end point events (cardiovascular death, myocardial infarction, stroke, hospitalization for unstable angina, and coronary revascularization) and in key secondary endpoint events (cardiovascular death, myocardial infarction and stroke) were comparable: $5.2 \%$ vs $4.2 \%$, Relative risk reduction [RRR] $(95 \% \mathrm{CI}) 0.79(0.61,1.03)$; and $3.6 \%$ vs $2.7 \%$, RRR (95\% CI) 0.73 (0.53, 1.01), respectively, in Asian patients, and 5.4\% vs 4.6\%, RRR (95\% CI) 0.86 (0.79, 0.93); and $3.4 \%$ vs $2.8 \%$, RRR $(95 \% \mathrm{CI}) 0.81(0.73,0.89)$, in others (both $p$ values for treatment interactions were not significant). Serious adverse event rates were similar amongst
Asian participants vs other races (11.8\% vs $12.5 \%$, respectively, per annum), and adverse events-related study drug discontinuations were low in both the Asian participants and other subjects ( $1.5 \%$ vs $2.1 \%$ per annum). The data further proved the efficacy and safety of the use of evolocumab in Asian subjects. Furthermore, there was no need to modify the evolocumab dose for Asians.

Evolocumab is quite effective in $\mathrm{HeFH}$ patients and yielded $60 \%$ reduction in LDL cholesterol, similar in magnitude to that observed in those without the disorder, indicating no association with the underlying genetic mutations of HeFH patients. ${ }^{18}$ The efficacy of evolocumab in HoFH treatment is variable, depending on the mutation status of the LDLR. Response to evolocumab is better in patients with 2 LDLR-defective mutations than in those with the presence of a single LDLR-negative mutation. HoFH patients with 2 LDLR-negative mutations does not respond to evolocumab at all and should not be treated with this class of medication. ${ }^{10}$

Regarding the mutation types of LDLR in Chinese population, one systematic review had reported 131 LDLR mutations in Chinese population. ${ }^{19}$ Most of the mutations were located in exon 4 of LDLR and approximately $60 \%$ of the mutations were missense mutations. The most frequent LDLR mutations were C329Y, H583Y, and A627T, which all had functional significance. ${ }^{19}$ In our previous review, total 143 different LDLR mutations had been identified in Taiwan, including 134 point mutations and 9 large rearrangements; the 5 most frequent mutations included APOB-R3527W, followed by LDLR mutations C329Y, H583Y, A627T and D90N. ${ }^{5}$ In a recent systematic review, the incidence rates of LDLR and APOB mutations were $82 \%$ and $9 \%$ respectively, and PCSK9 mutations were very rare in Chinese patients with $\mathrm{FH}^{20}$

In terms of the genotypes of $\mathrm{HoFH}$ patients, $41 \mathrm{HoFH}$ patients have been genetically diagnosed, including 9 true homozygous, 30 compound heterozygous and 2 double heterozygous, based on approximately 1000 consecutive sequencing of clinical FH patients in Taiwan. Furthermore, 4 out of the $41 \mathrm{HoFH}$ patients were actually LDLR negative/negative genotypes with untreated LDL cholesterol level greater than $800 \mathrm{mg} / \mathrm{dL}$, and were not expected to respond to evolocumab treatment, which would be at the very least partially effective in HoFH patients with other genotypes in the presence of at least one defective but functional LDLR allele. Therefore, HoFH patient genetic information is valuable and useful in the selection of patients for evolocumab treatment in clinical practice. 
Since March 1st 2018, HoFH treatment with evolocumab has been reimbursed by Taiwan's national health insurance, and homozygous genotype, proved by genetic testing, is required for reimbursement. In China, since December 28th 2018, evolocumab has been available as compassionate use for HoFH patients. About $30 \mathrm{HoFH}$ patients have been treated with evolocumab with variable LDL cholesterol lowering responses. As far as we know, there was no direct data about the appropriate dosage of evolocumab in Chinese HoFH patients. In a pharmacokinetics (PK) and pharmacodynamics (PD) study of evolocumab, there were no significant differences in the PK/PD relationship of its LDL cholesterol lowering effects between Caucasians and Asians. ${ }^{21}$ The current approval dosage of evolocumab for HoFH treatment were $420 \mathrm{mg}$ subcutaneously once a month in both China and Taiwan, which were the same recommended dosage as that in Caucasians. Therefore, although there were no direct evolocumab clinical trials in Chinese HoFH patients, its efficacy and safety in Chinese patients should be similar to that in different ethnic groups.

\section{Conclusion}

Evolocumab, a PCSK9 inhibitor, is effective and safe in severe hypercholesterolemia patients. Presently, there are no direct evidences of its use of in Chinese HoFH patients. However, based on the currently available genotyping results of Taiwanese, evolocumab should be effective and safe in approximately $90 \%$ of Chinese HoFH patients.

\section{Acknowledgments}

The authors thank Hsing-Yi Liu for her excellent technological assistance.

\section{Funding}

This work was funded by Taiwan's National Ministry of Science and Technology (Grant number, MOST105-2314B-075-040) and by Taipei Veterans General Hospital (Grant numbers, V105C-208 and V107C-078).

\section{Disclosure}

The authors report no conflicts of interest in this work.

\section{References}

1. Benn M, Watts GF, Tybjaerg-Hansen A, Nordestgaard BG. Familial hypercholesterolemia in the danish general population: prevalence, coronary artery disease, and cholesterol-lowering medication. J Clin Endocrinol Metab. 2012;97(11):3956-3964. doi:10.1210/jc.2012-1563
2. Marks D, Thorogood M, Neil HA, Humphries SE. A review on the diagnosis, natural history, and treatment of familial hypercholesterolaemia. Atherosclerosis. 2003;168(1):1-14. doi:10.1016/s0021-9150(02) 00330-1

3. Nordestgaard BG, Chapman MJ, Humphries SE, et al. Familial hypercholesterolaemia is underdiagnosed and undertreated in the general population: guidance for clinicians to prevent coronary heart disease: consensus statement of the European Atherosclerosis Society. Eur Heart J. 2013;34(45):3478-3490a. doi:10.1093/eurheartj/eht273

4. Catapano AL, Graham I, De Backer G, et al. ESC/EAS guidelines for the management of dyslipidaemias: the task force for the management of dyslipidaemias of the European Society of Cardiology (ESC) and European Atherosclerosis Society (EAS) developed with the special contribution of the European Association for Cardiovascular Prevention \& Rehabilitation (EACPR). Atherosclerosis. 2016;253:281-344. doi:10.1016/j. atherosclerosis.2016.08.018

5. Chiou KR, Charng MJ. Genetic diagnosis of familial hypercholesterolemia in Han Chinese. J Clin Lipidol. 2016;10(3):490-496. doi:10. 1016/j.jacl.2016.01.009

6. Marais AD, Blom DJ. Recent advances in the treatment of homozygous familial hypercholesterolaemia. Curr Opin Lipidol. 2013;24 (4):288-294. doi:10.1097/MOL.0b013e32836308bc

7. Pang J, Chan DC, Hu M, et al. Comparative aspects of the care of familial hypercholesterolemia in the "Ten Countries Study". J Clin Lipidol. 2019;13(2):287-300. doi:10.1016/j.jacl.2019.01.009

8. Sabatine MS, Giugliano RP, Keech AC, et al. Evolocumab and clinical outcomes in patients with cardiovascular disease. $N$ Engl $J$ Med. 2017;376(18):1713-1722. doi:10.1056/NEJMoa1615664

9. Stein EA, Honarpour N, Wasserman SM, Xu F, Scott R, Raal FJ. Effect of the proprotein convertase subtilisin/kexin 9 monoclonal antibody, AMG 145, in homozygous familial hypercholesterolemia. Circulation. 2013;128(19):2113-2120. doi:10.1161/CIRCULATION AHA.113.004678

10. Raal FJ, Honarpour N, Blom DJ, et al. Inhibition of PCSK9 with evolocumab in homozygous familial hypercholesterolaemia (TESLA Part B): a randomised, double-blind, placebo-controlled trial. Lancet. 2015;385(9965):341-350. doi:10.1016/S0140-6736 (14)61374-X

11. Raal FJ, Hovingh GK, Blom D, et al. Long-term treatment with evolocumab added to conventional drug therapy, with or without apheresis, in patients with homozygous familial hypercholesterolaemia: an interim subset analysis of the open-label TAUSSIG study. Lancet Diabetes Endocrinol. 2017;5(4):280-290. doi:10.1016/S22138587(17)30044-X

12. Santos RD, Hovingh GK, Blom D, et al. Long-term evolocumab treatment in homozygous and severe heterozygous familial hypercholesterolemia: the TAUSSIG trial. J Am Coll Cardiol. 2019;73(9 Suppl 1):1715. doi:10.1016/S0735-1097(19)32321-6

13. Hirayama A, Honarpour N, Yoshida M, et al. Effects of evolocumab (AMG 145), a monoclonal antibody to PCSK9, in hypercholesterolemic, statin-treated Japanese patients at high cardiovascular risk-primary results from the phase 2 YUKAWA study. Circ J. 2014;78(5):1073-1082. doi:10.1253/circj.cj-140130

14. Hirayama A, Yamashita S, Inomata H, et al. One-year efficacy and safety of evolocumab in Japanese patients - a pooled analysis from the open-label extension OSLER studies. Circ J. 2017;81(7):10291035. doi:10.1253/circj.CJ-16-1016

15. Lorenzatti AJ, Eliaschewitz FG, Chen Y, et al. Randomised study of evolocumab in patients with type 2 diabetes and dyslipidaemia on background statin: primary results of the BERSON clinical trial. Diabetes Obes Metab. 2019;21(6):1455-1463. doi:10.1111/ dom. 13680 
16. Chen Y, Yuan Z, Lu J, et al. Randomized study of evolocumab in patients with type 2 diabetes and dyslipidaemia on background statin: pre-specified analysis of the Chinese population from the BERSON clinical trial. Diabetes Obes Metab. 2019;21(6):1464-1473. doi:10.1111/dom.13700

17. Keech AC, Sever PS, Jiang L, et al. Efficacy and safety of long-term evolocumab use in Asian versus other subjects: the FOURIER trial. $J$ Am Coll Cardiol. 2019;73(9 Suppl 1):189. doi:10.1016/S0735-1097 (19)30797-1

18. Raal FJ, Stein EA, Dufour R, et al. PCSK9 inhibition with evolocumab (AMG 145) in heterozygous familial hypercholesterolaemia (RUTHERFORD-2): a randomised, double-blind, placebo-controlled trial. Lancet. 2015;385(9965):331-340. doi:10.1016/S0140-6736(14) 61399-4
19. Jiang L, Sun LY, Dai YF, Yang SW, Zhang F, Wang LY. The distribution and characteristics of LDL receptor mutations in China: a systematic review. Sci Rep. 2015;5:17272. doi:10.1038/ srep 17272

20. Peng J, Wu X, Wang S, et al. Familial hypercholesterolemia in China half a century: a review of published literature. Atheroscler Suppl. 2019;36:12-18. doi:10.1016/j.atherosclerosissup.2019.01.003

21. Wang C, Zheng Q, Zhang M, Lu H. Lack of ethnic differences in the pharmacokinetics and pharmacodynamics of evolocumab between Caucasian and Asian populations. Br J Clin Pharmacol. 2019;85 (1):114-125. doi:10.1111/bcp. 13767

\section{Publish your work in this journal}

Therapeutics and Clinical Risk Management is an international, peerreviewed journal of clinical therapeutics and risk management, focusing on concise rapid reporting of clinical studies in all therapeutic areas, outcomes, safety, and programs for the effective, safe, and sustained use of medicines. This journal is indexed on PubMed Central, CAS,
EMBase, Scopus and the Elsevier Bibliographic databases. The manuscript management system is completely online and includes a very quick and fair peer-review system, which is all easy to use. Visit http://www.dovepress.com/testimonials.php to read real quotes from published authors 